\title{
Globe
}

Revue internationale d'études québécoises

\section{Yvon Roberge : Histoire des TCA au Québec. Connaître le passé pour façonner l'avenir, Fides, Montréal, 2008}

\section{Serge Denis}

Volume 12, numéro 2, 2009

URI : https://id.erudit.org/iderudit/1000721ar

DOI : https://doi.org/10.7202/1000721ar

Aller au sommaire du numéro

Éditeur(s)

Globe, Revue internationale d'études québécoises

ISSN

1481-5869 (imprimé)

1923-8231 (numérique)

Découvrir la revue

Citer ce compte rendu

Denis, S. (2009). Compte rendu de [Yvon Roberge : Histoire des TCA au Québec. Connaître le passé pour façonner l'avenir, Fides, Montréal, 2008]. Globe, 12(2), 230-235. https://doi.org/10.7202/1000721ar d'utilisation que vous pouvez consulter en ligne.

https://apropos.erudit.org/fr/usagers/politique-dutilisation/ 
Quant à la toilette du texte, elle est généralement soignée, bien que la mise en page ne soit pas uniformisée, notamment en ce qui concerne la structuration des articles sur le plan des intertitres (voir les contributions d'Audrée Descheneaux et de Caroline Durand). Dernier point par lequel je soulève un problème général et qui n'est donc pas une critique visant $\mathrm{La}$ chanson francophone engagke: comme pour toute publication sur la chanson, le lecteur intéressé souhaiterait avoir à sa disposition les enregistrements des chansons à l'étude ${ }^{13}$ ou d'autres éléments facilitant la compréhension du propos, comme par exemple les paroles d'une chanson dans l'annexe de l'article (voir celui de Luc Bellemare), des extraits de partition ou des schémas musicaux (tels que proposés dans l'article d'Audrée Descheneaux).

Dans l'ensemble, les réflexions fort intéressantes de ces jeunes chercheurs sur "la chanson francophone engagée " témoignent non seulement de la diversité de ce type de chanson mais également de la pluralité des approches et des méthodes d'analyse permettant de comprendre la "chanson" comme un fait social et de la saisir en même temps comme un objet d'étude sérieux. C'est une publication engagée qui répond en quelque sorte à la problématique que les auteurs se proposent d'explorer par divers moyens.

Andrea Oberhuber Université de Montréal

\section{Yvon Roberge}

Histoire des TCA au Québec.

Connaître le passé pour façonner l'avenir

Fides, Montréal, 2008.

Yvon Roberge est présenté en quatrième de couverture comme un "observateur privilégié" de l'activité et des mœurs du syndicat des Travailleurs canadiens de l'automobile (autrefois les Travailleurs unis de l'automobile), ayant "milité activement» aux TCA durant une trentaine d'années. Il veut manifestement avec ce livre offrir une histoire éducative de son

$+4$

13. Je sais bien évidemment que l'ajout d'un disque compact pose le problème des droits d'auteur et qu'il est pratiquement inimaginable de pouvoir financer ces droits qui protègent la propriété intellectuelle des créateurs. Il s'agit simplement de formuler une fois de plus ce desideratum. 
syndicat, notamment pour la nouvelle génération de ses membres. Mais ce faisant, son récit s'arrête à des épisodes marquants du développement sociopolitique et socioéconomique du Québec contemporain, auxquels le syndicat des travailleurs et travailleuses de l'automobile a souvent été associé (et qu'il a servi à façonner), de sorte qu'il pourra aussi intéresser les chercheurs et les spécialistes en sciences sociales de même que les militants et les militantes d'autres organismes populaires. Le livre compte sept chapitres complétés par dix annexes, dont une reconstitution chronologique de l'évolution du mouvement ouvrier québécois, qui va de ses origines à aujourd'hui. De bout en bout, le texte se déroule comme une longue description des faits et gestes du syndicat, de ses structures également, depuis (pour l'essentiel) les années 1960, une description attentive et détaillée, presque hagiographique parfois, un peu à l'instar d'un document interne par lequel une organisation viserait précisément à consigner sa propre mémoire (d'ailleurs, le livre est préfacé par Luc Desnoyers, qui a été directeur des TCA-Québec de 1995 à 2008). Il s'agit d'un type d'exposé qui alourdit le texte à l'occasion, trop détaillé sur certains aspects et insuffisamment analytique sur d'autres; cela dit, cette démarche devient fort éloquente aussi, et révélatrice, par les événements et les péripéties mêmes qu'elle met en exergue. Par exemple, la place majeure que tiennent la politique et les préoccupations relevant de la réalité nationale du Québec dans le texte de Roberge, couvrant concrètement plus de la moitié du traitement de son sujet, exprime en quelque sorte par elle-même des caractéristiques incontournables de la conscience sociale des militants de la génération d'Yvon Roberge et des axes forts de la dynamique du développement syndical durant les décennies qu'ouvre la "Révolution tranquille». On notera, par ailleurs, quelques erreurs dans le texte, de fait ou d'inattention (à la page 13, par exemple, il est écrit "CTC " plutôt que "CCT" pour l'année 1940), mais elles ne diminuent pas l'intérêt qu'il peut représenter.

Les deux premiers chapitres reviennent sur l'histoire du syndicalisme au Canada et au Québec, à grands traits jusqu’à la création du syndicat en 1936 et ses premières années d'existence, qui sont étudiées de façon plus précise. C'est en 1946 que la première unité d'accréditation est gagnée au Québec; mais le syndicat y sera peu présent jusqu'aux années 1960 , quand ses grandes campagnes chez GM à Sainte-Thérèse, puis United Aircraft à Longueuil (au sud de Montréal), etc. en viendront à ponctuer pour une large part la vie sociale de la province. Les travailleurs de l'automobile connaissent en effet un impressionnant essor durant les décennies 1960-1970 et 1970-1980 au Québec. Les syndiqués canadiens 
furent membres de l'union des Travailleurs Unis de l'automobile pendant près d'un demi-siècle, formant la "région 7" - appelée par la suite "région canadienne" - de cette grande organisation dont le centre de gravité, comme celui de l'industrie de l'automobile en Amérique du Nord, se situait aux États-Unis. En 1986, il y eut séparation à l'amiable et formation du syndicat indépendant des Travailleurs canadiens de l'automobile.

Ce divorce s'inscrivait incontestablement dans la continuité des demandes d'une autonomie plus grande de la section canadienne au sein des TUA, demandes pour une part liées à l'adoption des orientations spécifiques du mouvement ouvrier canadien, souvent différentes au fil des décennies de celles mises de l'avant aux États-Unis mais auxquelles les syndiqués de l'automobile au Canada tenaient à se rallier (l'exemple est donné d'une campagne de réduction des heures de travail), et à une volonté évidente d'être davantage maître chez soi. Comme le syndicat des TUA se pliait généralement à cette demañde d'autonomie, la séparation n'était institutionnellement pas inéluctable; mais il est certain que des divergences plus profondes se manifestèrent durant les dernières années de la vie en commun. Ainsi, le syndicalisme canadien refusa de manière unie, dès le tournant des années 1980, les politiques de concessions lors du renouvellement des conventions collectives qu'acceptèrent de leur côté les TUA américains. Il n'était plus possible, dès lors, de négocier avec les trois grands de l'automobile sur la base des mêmes critères, et le divorce apparut logique à l'une et l'autre parties.

Sur cette question de la présence des syndicats américains au Canada depuis le XIX siècle, je voudrais souligner cependant, contrairement à ce que semble suggérer le texte, que leur arrivée ne fut pas d'abord le produit d'une volonté de domination de leur part. Les causes sont plus complexes, comme l'ont montré déjà des historiens: elles relèvent, par exemple, de ce que les marchés du travail aux États-Unis et au Canada n'étaient alors pas pleinement différenciés, que l'état du développement technologique et des métiers était équivalent d'un côté et de l'autre de la frontière, deux éléments qui entraînaient beaucoup de va-et-vient des salariés entre les deux pays, cependant que certains groupes de travailleurs canadiens ont aussi invité directement des syndicats américains (plus solides à l'époque que ceux du Canada) à venir leur prêter main-forte.

Dans le troisième chapitre, intitulé "Le développement démocratique ", Yvon Roberge s'arrête à la problématique de la présence dans les TUA-TCA de milliers de syndiqués québécois à partir des années 1960, de ce que pouvait signifier pour eux la démocratie dans leur organisation et du 
rôle qu'ils voulaiènt voir leur syndicat jouer dans la société québécoise. Très vite, ils demandent la mise sur pied d'un conseil québécois distinct. Selon Roberge, s'il est sûr que cette volonté découlait de ce que les grandes rencontres pancanadiennes se déroulaient dans une langue, l'anglais, qui n'était pas celle des Québécois, "il y avait plus que cela" : la quête de "l'identité québécoise" amenait aussi les syndiqués à désirer "leurs propres structures. Ils voulaient pouvoir débattre de leurs problèmes et y trouver leurs solutions. Ils voulaient révéler au monde leur vision de la société d'alors et du futur". Ils devaient donc compter sur les mêmes "moyens que ceux fournis à leurs confrères de langue anglaise. Ils se sentaient leurs égaux, sans avoir les outils pour le prouver" (p. 29). Roberge associe fort justement le sens de cette requête à l'histoire de la société québécoise des décennies 1960-1980, engagée dans ce qu'il définit comme une "quête d'identité" (dont il voit une expression directe dans la création du Parti québécois et dans l'action de René Lévesque).

Sur le même terrain, soulignons que la grève des travailleurs de l'automobile chez GM à Sainte-Thérèse en 1970 marquera une étape majeure dans le rayonnement de la revendication du français comme langue de travail (et du contrat collectif) au Québec. Les TUA avaient alors lancé cette grève contre General Motors dans toute l'Amérique du Nord; si les revendications étaient sensiblement les mêmes pour le Canada et les États-Unis, au Québec, explique Roberge, il y avait un élément particulier: les travailleurs exigeaient que le français devienne la langue de leur travail (les communications internes à l'usine se faisaient en anglais, par exemple), ce que la compagnie refusa obstinément. Toujours en grève pour cette revendication deux mois après que les syndiqués des États-Unis et du Canada anglais aient repris le travail, les syndiqués de Sainte-Thérèse reçurent la visite de René Lévesque, qui leur déclara: "ce n'est pas à 2000 Québécois courageux de porter le Québec sur leurs épaules. Retournez au travail, nous autres on va s'occuper du problème". Le mouvement de Sainte-Thérèse constitua ainsi, explique avec raison l'auteur, une impulsion directe à l'adoption plus tard de la Loi 101 (pour cet épisode, voir le quatrième chapitre, p. 49-52). Limpact de la grève chez United Aircraft en 1974-1975 allait s'avérer à terme tout aussi décisif: cette grève fut en effet à l'origine de la Loi 45, amendant le Code du travail et qu'adoptera également le futur gouvernement du Parti québécois. Les amendements sont ceux qui interdiront au Québec «l'utilisation de briseurs de grève, qui [garantiront] le rappel au travail des grévistes en priorité sur toute autre personne (à la suite d'un arrêt de travail) et [rendront] obligatoire la formule Rand" dans les conventions collectives (p. 85). 
Évidemment, les TUA-TCA québécois ne constituent pas la source unique de ces grandes transformations. Mais il est vrai que leurs actions ont constitué autant d'impulsions importantes à leur réalisation, dont le rappel contribue à mieux comprendre certaines dimensions du développement sociopolitique du Québec et des réalités partisanes durant les années 1970. Il faut cependant faire ici deux précisions: les TUA-TCA canadiens ne se sont pas opposés à la création d'un conseil québécois spécifique, dont l'existence est officialisée depuis 1985 dans les statuts du syndicat, mais qui se réunissait avec son approbation depuis que la demande en avait été faite; la section du Québec a soutenu le "oui " référendaire en 1980 et en 1995, alors qu'avant le référendum de 1980 , le syndicat de l'ensemble du Canada a adopté une résolution de soutien au droit à l'autodétermination du Québec, ce qui n'est pas rien.

Il convient maintenant de se pencher expressément sur le traitement de l'action politique du syndicat au Québec et au Canada anglais. Traditionnellement, les TUA-TCA appuyaient le Nouveau Parti démocratique (NPD) à l'extérieur du Québec, aidaient à son financement et des sections locales du syndicat en étaient collectivement membres; au Québec, l'appui au Parti québécois est venu très rapidement. Roberge n'établit pas de différences entre ces deux types d'appui, c'est-à-dire entre l'appui à un parti que le mouvement ouvrier a contribué à créer et auquel il est formellement attaché (le NPD) et l'appui à un parti dont on apprécie le programme, mais qui ne relève pas du syndicalisme. À mon avis, il y a là un creux dans l'analyse pour qui s'intéresse spécifiquement à l'histoire des mouvements ouvriers, mais il est compréhensible du fait que le parti de René Lévesque apparut effectivement comme un parti nettement à la gauche des autres formations présentes à l'Assemblée nationale et en raison du caractère socialement contestataire qu'avait acquis le nationalisme durant les années 1960.

Cela dit, sur près de quarante pages (p. 91-130), l'auteur présente dans le cinquième chapitre la crise de leur action politique qu'il considère, à l'instar des TCA eux-mêmes, aujourd'hui majeure et il relate la longue discussion qu'ils ont menée dans leurs rangs, de 1999 à 2003, sur l'avenir de "la politique ouvrière au $21^{\mathrm{e}}$ siècle». Mais Roberge se contente à cet égard de reprendre sur le fond les explications du syndicat quant à la nécessité de cet exercice et de citer (un peu pesamment) ses textes. Ici, le compte rendu aurait gagné à inclure une mise en perspective analytique plus large. Les TUA-TCA avaient en effet dépassé historiquement le type d'engagement politique que pratiquait le syndicat aux États-Unis: ils avaient contribué à créer un parti, alors que les Américains s'en étaient tenus à la vieille politique dite de la non- 
partisanerie (adopter des orientations et voir quels partis ou quels candidats s'en rapprochent le plus, puis le faire savoir, encourager ses membres à s'investir dans le parti de leur choix et à y faire valoir les préférences du syndicat pour la plate-forme électorale et la sélection de candidats - les TUA américains ne se sont finalement investis que dans le Parti démocrate -, encourager même les membres à intervenir conjointement à cette fin dans le parti où ils se retrouvent à plusieurs, etc.), même si elle a pu être fort active. La crise de l'action politique des TCA s'inscrit dans la crise de la socialdémocratie mondiale, vécue ici par rapport aux politiques du NPD (singulièrement du NPD-Ontario à l'époque du gouvernement de Bob Rae entre 1990 et 1995). Du fait de leur histoire et de leur maturité politiques, on aurait pu s'attendre à ce que la discussion au sein des TCA soit plus créative, se nourrisse du contenu des démarches réflexives semblables à la sienne en Europe du Nord et de l'Ouest, etc., y contribuent aussi. Mais de fait, c'est à un simple retour à la non-partisanerie d'un type bien traditionnel que le syndicat convie dorénavant ses membres au Canada. Une conclusion très décevante. Par ailleurs, il semble que le conseil québécois du syndicat entende maintenant susciter la participation à la tendance de gauche constituée au sein du PQ et appelée Syndicalistes et progressistes pour un Québec libre (SPQ libre), orientation se situant davantage que cela n'est le cas au Canada anglais, me semble-t-il, dans la suite de la politique distincte qu'il poursuivait jusqu'ici.

Le syndicat des travailleurs de l'automobile a joué depuis sa formation un rôle d'avant-garde dans le mouvement ouvrier, aux États-Unis notamment mais aussi au Canada. Ses activités ont profondément influencé la vie sociale de l'Amérique du Nord et ont été à l'origine de plusieurs des acquis ouvriers les plus marquants; il a été pour beaucoup d'historiens et de commentateurs le syndicat de masse le plus progressiste du continent. Il est pratiquement en train de s'effondrer pourtant, avec son industrie de base, au moment où j'écris cette recension, et des dizaines de milliers de ses membres, passés et présents, perdent chacun le fruit du labeur de toute une vie. $\mathrm{Ce}$ serait une fin bien triste si les événements devaient suivre ainsi jusqu'au bout la tangente dans laquelle ils sont présentement engagés. En tout état de cause, il apparaît que le rôle primordial qu'ont tenu les travailleurs de l'automobile dans l'histoire sociale de l'Amérique du Nord soit dorénavant, et malheureusement, révolu.

Serge Denis

Université d'Ottawa 